\title{
Precision measurement of the luminosity in the ATLAS experiment
}

\author{
Vincent Hedberg* \\ Department of physics, University of Lund, Sweden \\ E-mail: Vincent.Hedberg@cern.ch \\ on behalf of the ATLAS collaboration
}

\begin{abstract}
A precision luminosity measurement is of critical importance for the ATLAS physics program, both for searches for new physics as well as for precision measurements of Standard Model crosssections. The calibration of the luminosity is based on so-called van der Meer scans. The calibration determines the convolved beam sizes in the vertical and horizontal directions, and together with the precise knowledge of the beam current a luminosity calibration can be determined. Based on this analysis ATLAS has determined the luminosity of $p p$ collisions with an uncertainty of $\delta L / L=3.7 \%$ for the 2011 data at $\sqrt{s}=7 \mathrm{TeV}$.
\end{abstract}

The 2011 Europhysics Conference on High Energy Physics, EPS-HEP 2011,

July 21-27, 2011

Grenoble, Rhône-Alpes, France

\footnotetext{
* Speaker.
} 


\section{Introduction}

An accurate measurement of the luminosity is an essential part of the ATLAS physics program, both for Standard Model cross-section measurements, but also in searches for new physics when background and search sensitivity is estimated. The ATLAS experiment is determining luminosity by measuring the rate of inelastic interactions. This rate is calibrated by so-called beam separation scans, also known as van der Meer scans, in which the rate is measured as the two LHC beams are moved apart. The statistical error in a luminosity measurement using inelastic interactions is almost always negligible and so the main question in this type of luminosity analysis is what systematic accuracy can be achieved.

\section{The luminosity measurement}

ATLAS has several detectors and methods for measuring luminosity [1]. All of these are sampling the rate of inelastic interactions. In principle a process with a known theoretical cross section, such as W- or Z-production, can also be used for luminosity measurement but that would preclude ATLAS from measuring these cross sections. The uncertainty in the theoretical cross sections for W- and Z-production is also larger than what can be achieved by a calibration using a beam-separation scan.

At a collider where there is rarely no more than one interaction for every bunch crossing, the luminosity is proportional to the rate of triggers from a detector that is sensitive to inelastic events. The luminosity at the LHC is, however, so high that there are typically many inelastic interactions every time a pair of proton bunches cross each other in ATLAS. For a sample of bunch crossings the distribution of the number of interactions is a Poisson distributed quantity with a mean that can be denoted by $\mu$. A particular detector and method will measure a visible number of interactions $\left(\mu^{v i s}\right)$ that depends on the method's efficiency and acceptance $(\varepsilon)$ to detect a single interaction $\left(\mu^{v i s}=\varepsilon \mu\right)$. The name of the game is to measure $\mu^{v i s}$ with as high accuracy as possible during a time period called a luminosity block (typically one minute long) and then calculate the luminosity from that:

$$
\mathscr{L}_{L B}=\frac{f_{L H C}}{\sigma_{\text {inel }}} \sum_{j=1}^{n_{b}} \mu_{j}=\frac{f_{L H C}}{\sigma^{v i s}} \sum_{j=1}^{n_{b}} \mu_{j}^{v i s}
$$

where $\mathscr{L}_{L B}$ is the average luminosity during a luminosity block, $f_{L H C}$ is the LHC revolution frequency, $\sigma_{\text {inel }}$ is the total inelastic cross section, $n_{b}$ is the number of bunch pairs colliding in ATLAS and $\sigma^{v i s}$ is the visible cross section, i.e., it is the inelastic cross section times the efficiency and acceptance of the luminosity method that is being used $\left(\sigma^{v i s}=\varepsilon \sigma_{\text {inel }}\right)$.

ATLAS has two detectors, LUCID and BCM, that can measure $\mu^{\text {vis }}$ online for individual bunchcrossings. LUCID consists of two gas Cerenkov detectors that surround the beampipe on both sides of the interaction point (IP). The BCM detector has four diamond detectors on each side of the IP. The read-out of these eight detectors is split in $2+2$ horizontal and $2+2$ vertical detectors called $\mathrm{BCM} \mathrm{V}$ and BCM H. Both LUCID and BCM are determining $\mu^{v i s}$ by measuring the fraction of bunch-crossings in a luminosity block with a signal in the detectors under certain conditions. The simplest condition is only that at least one detector element has a signal. This is called "Event OR" counting. In "Event AND" counting it is instead required that there is at least one signal on both 
sides of the IP. Formulas have been derived that relate the measured fractions to $\mu^{v i s}$ under these conditions and under two assumptions [1]. Firstly it is assumed that the number of interactions in a luminosity block follows a Poisson distribution. Secondly that the probability to have no signal in multiple interactions can be calculated from the probability to have no signal in a single interaction. The second assumption is not always perfectly correct since small signals below the discriminator thresholds can add up in multiple interactions and increase the efficiency of the method.

ATLAS is also using two calorimeters called the Forward Calorimeter (FCal) and the Hadronic Tile Calorimeter (Tile) to measure the luminosity. These calorimeters monitor the currents drawn in the different parts of the detectors due to inelastic collisions. They do not have the possibility to measure the luminosity for an individual bunch-crossing but this is not necessary as long as the measured current is perfectly proportional to the luminosity. A comparison of the average $\mu$-values measured by different detectors and methods is shown in Figure 1 as a function of $\mu$. Most points are within a band of $\pm 1 \%$ and so this has been assigned as a preliminary uncertainty in the $\mu$ measurement. Another systematic uncertainty is due to the long term stability of the detectors. A comparison of the average luminosity measured by different detectors and methods for different ATLAS data-taking runs is depicted in Figure 2. Most of the data are again within $\pm 1 \%$ which have been used as the systematic errors for longtime stability.
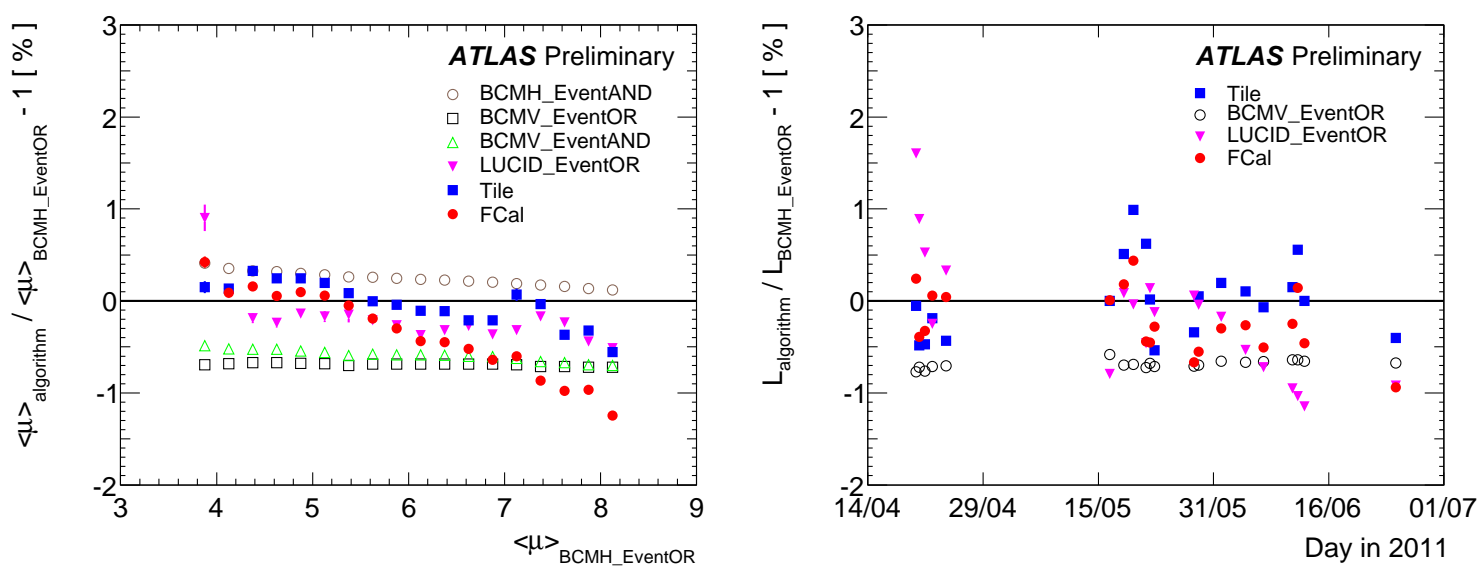

Figure 1: Fractional deviation in the average num- Figure 2: Fractional deviation in the integrated luber of interactions per bunch crossing $\langle\mu\rangle$ (averaged minosity obtained using different algorithms with reover all bunch crossings), obtained using different al- spect to the BCMH_EventOR value as a function of gorithms with respect to the BCMH_EventOR value time [1]. Each point shows the average deviation as a function of $\langle\mu\rangle[1]$. Statistical uncertainties per for a single ATLAS run. Statistical uncertainties per point are negligible. point are negligible.

\section{The van der Meer scan calibration}

If a detector in ATLAS is measuring the interaction rate while the beams are being separated in the horizontal (x) and vertical direction (y) then the width of the two scan curves $\left(\Sigma_{x}\right.$ and $\left.\Sigma_{y}\right)$ can be used to estimate the luminosity. One can show that the maximum luminosity for a colliding bunch pair $\left(\mathscr{L}^{\text {peak }}\right)$ can be calculated from the overlap integral of the transverse proton density functions $\left(\rho_{1}(x, y)\right.$ and $\left.\rho_{2}(x, y)\right)$ of the two beams 1 and 2 and if these density functions are not correlated in 
$\mathrm{x}$ and $\mathrm{y}$, the integral can be obtained from the scan widths:

$$
\mathscr{L}^{\text {peak }}=n_{p 1} n_{p 2} \mathscr{L}_{\text {spec }}^{\text {peak }}=f_{L H C} n_{p 1} n_{p 2} \int \rho_{1}(x, y) \rho_{2}(x, y) d x d y=f_{L H C} n_{p 1} n_{p 2} \frac{1}{2 \pi \Sigma_{x} \Sigma_{v}}
$$

where $\mathscr{L}_{\text {spec }}^{\text {peak }}$ is the so-called specific luminosity and $n_{p 1(2)}$ is the number of protons in each of the two colliding bunches. Combining equation 2.1 with 3.1 gives an expression for the visible cross section:

$$
\sigma^{v i s}=2 \pi \frac{\mu_{p e a k}^{v i s} \Sigma_{x} \Sigma_{y}}{n_{p 1} n_{p 2}}
$$

The relative systematic error in the measurement of $\mu_{\text {peak }}^{\text {vis }} \Sigma_{x} \Sigma_{y}$ is estimated to be $\pm 1.5 \%$ (including the uncertainty in the assumption of no correlation in $\mathrm{x}$ and $\mathrm{y}$ ). Figure 3 shows the excellent agreement in the measurement of $\mathscr{L}_{\text {spec }}^{\text {peak }}\left(\sim 1 / \Sigma_{x} \Sigma_{y}\right)$ for each BCID when using the LUCID and BCM de-

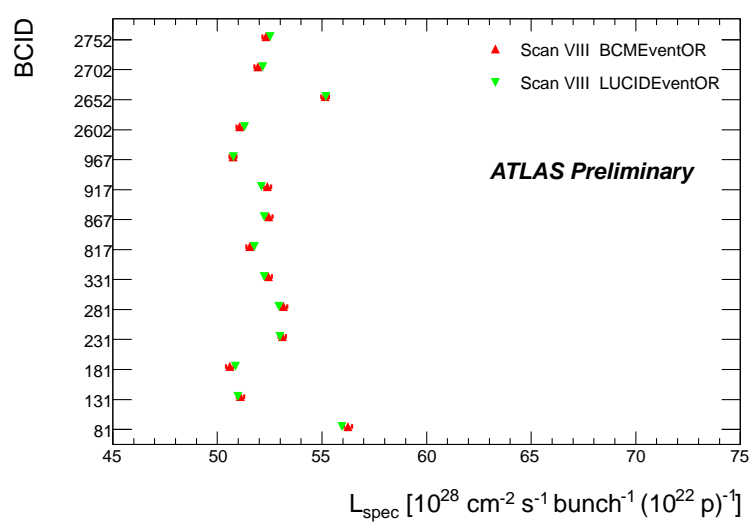

Figure 3: Specific luminosity determined by BCM H and LUCID for the 14 different colliding bunch crossings during a scan in 2011 [1]. Statistical uncertainties are the size of the points. tectors (differences of $\mathscr{L}_{\text {spec }}^{\text {peak }}$ between different bunches are expected).

The final and largest systematic error is due to the measurement of the number of protons $\left(n_{p 1} n_{p 2}\right)$. This is done by a pair of DC current transformers (DCCT) that measure the total LHC current and a pair of fast beam current transformers (FBCT) that measure the fraction of the total current in each bunch. The following expression is used to estimate the number of protons in a bunch j:

$$
n_{p j}=\left(\alpha\left(S^{D C C T}-S_{\text {baseline }}^{D C C T}\right)-N_{\text {ghostcharge }}^{D C C T}\right) \frac{S_{j}^{F B C T}}{\sum_{i=1}^{n_{b}} S_{i}^{F B C T}}
$$

where $\alpha$ is a calibrated scale factor, $S^{D C C T}$ is the uncalibrated DCCT measurement of the total number of protons which is corrected for a non-zero baseline and ghost charge and $S^{F B C T}$ is the measurement of the FBCT. The systematic error in the DCCT measurement of the product of the protons in both beams is estimated to be $\pm 2.7 \%$ while the error in the FBCT measurement is estimated to be $\pm 1.6 \%$. This results in a total error in the proton bunch product of $\pm 3.1 \%$ [1].

\section{Summary}

The preliminary total error in the measurement of the luminosity by ATLAS in 2011 is $\pm 3.7 \%$ and consists of the following four main components:

- Calibration error $\left(\sigma^{\text {vis }}\right): \pm 3.4 \%$

- $\mu$ determination: $\pm 1.0 \%$
- Detector stability: $\pm 1.0 \%$

- Background subtraction: $\pm 0.2 \%$

\section{References}

[1] "Luminosity Determination in pp Collisions at $\sqrt{s}=7$ TeV using the ATLAS Detector in 2011", ATLAS note ATLAS-CONF-2011-116 by the ATLAS collaboration and references therein. http://cdsweb.cern.ch/record/1376384 